\title{
BMJ Open Future Care Planning for patients approaching end-of-life with advanced heart disease: an interview study with patients, carers and healthcare professionals exploring the content, rationale and design of a randomised clinical trial
}

Martin A Denvir, ${ }^{1}$ Gill Highet, ${ }^{2}$ Shirley Robertson, ${ }^{1}$ Sarah Cudmore, ${ }^{1}$ Janet Reid, ${ }^{1}$ Andrea Ness, ${ }^{1}$ Karen Hogg, ${ }^{3}$ Christopher Weir, ${ }^{4}$ Scott Murray, ${ }^{5}$ Kirsty Boyd ${ }^{2}$

To cite: Denvir MA, Highet G, Robertson S, et al. Future Care Planning for patients approaching end-of-life with advanced heart disease: an interview study with patients, carers and healthcare professionals exploring the content, rationale and design of a randomised clinical trial. BMJ Open 2014;4:e005021. doi:10.1136/bmjopen-2014005021

- Prepublication history and additional material is available. To view please visit the journal (http://dx.doi.org/ 10.1136/bmjopen-2014005021)

Received 7 February 2014 Accepted 27 June 2014

CrossMark

For numbered affiliations see end of article.

Correspondence to Dr Martin A Denvir; martin.denvir@ed.ac.uk

\section{ABSTRACT}

Objective: To explore the optimal content and design of a clinical trial of an end-of-life intervention for advanced heart disease with patients, carers and healthcare professionals.

Design: Qualitative interview and focus group study.

Setting: Community and hospital-based focus groups and interviews.

Participants: Stable community-dwelling patients, informal carers (PC, $n=15)$ and primary and secondary care based healthcare professionals (HCP, $n=11$ ).

Results: PC highlighted fragmentation of services and difficulty in accessing specialist care as key barriers to good care. They felt that time for discussion with HCP was inadequate within current National Health Service (NHS) healthcare systems. HCP highlighted uncertainty of prognosis, explaining mortality risk to patients and switching from curative to palliative approaches as key challenges. Patient selection, nature of the intervention and relevance of trial outcomes were identified by HCP as key challenges in the design of a clinical trial.

Conclusions: PC and HCP expressed a number of concerns relevant to the nature and content of an endof-life intervention for patients with advanced heart disease. The findings of this study are being used to support a phase II randomised clinical trial of Future Care Planning in advanced heart disease.

\section{INTRODUCTION}

Patients with cancer have well-developed palliative care services while patients with advanced heart disease do not as highlighted in two major reports by The Department of Health in England and Wales ${ }^{1}$ and Scottish Government Action Plan 'Living and Dying

\section{Strengths and limitations of this study}

- This qualitative interview study has provided a $360^{\circ}$ perspective from patients, carers and healthcare professionals on the content, nature and mode of delivery of an intervention that could be tested in a clinical trial and that could impact on quality of life.

- The findings suggest that a randomised (early vs delayed) protocol is broadly acceptable, that clinical prognostic scores could be used to identify eligible patients in the hospital setting, that care needs should also be incorporated into the eligibility criteria and that the intervention should include components that address the current gaps in high-quality holistic care (as identified by patients and their carers).

- The relatively small number of patients and healthcare professionals participating in the study and the fact that all patients were stable in a community setting may have impacted on the findings.

- Engaging patients and carers in the rationale, content and design of a randomised clinical trial is challenging and requires careful design and planning.

Well'. ${ }^{2}$ These documents promote the provision of care in the last year of life that is person-centred regardless of diagnosis. The recent National Health Service (NHS) quality Improvement Scotland Clinical Standards for Heart Disease recommend a palliative care assessment in all forms of advanced heart disease. ${ }^{3}$ Recent publications relating to end-of-life (EOL) care in heart disease have focused on congestive heart 
failure (CHF) but coronary disease and valvular heart disease commonly coexist in patients with CHF, so an integrated approach to all end-stage heart diseases is appropriate.

We recently explored ways of identifying patients who are approaching EOL in an acute cardiology ward. Using the Gold Standards Framework criteria and validated prognostic tools we demonstrated that most patients with advanced heart failure ${ }^{4}$ and a lesser proportion with acute coronary events ${ }^{5}$ have a very limited prognosis despite optimal evidence-based care. Poor prognosis is a marker of lower quality of life, increased hospitalisation, multimorbidity ${ }^{6}$ and is an indirect marker of increasing patient needs. There are wellvalidated prognostic tools for patients with $\mathrm{CHF}^{7}$ and acute coronary syndrome (ACS) ${ }^{8}$ Once a patient with a poor prognosis is identified, this should ideally be followed by an evidence-based intervention ${ }^{9-11}$ that could improve quality of life for the patient and their family. In keeping with palliative care models, ${ }^{12}$ this intervention should be patient-focused and should address individual needs. Ideally the intervention should integrate patient preferences with clinical priorities using 'shared decision-making'. ${ }^{13}$ From these discussions, a Future Care Plan (FCP) may be derived and written in terms that the patient understands. The FCP should contain a clinical plan of how to manage acute events of deteriorating health with mechanisms to inform out-of-hours services and maintain continuity of care. The plan should be reviewed regularly in the context of the patient's evolving multidimensional needs. Such an intervention could be initiated by the patient's cardiologist and delivered by a specialist heart disease nurse working in partnership with the primary care team and palliative care specialists.

Trials of palliative care are recognised to be extremely difficult to design and implement. ${ }^{14}$ One previous randomised trial suggested that routine palliative care in addition to normal oncological care could improve quality of life in people with lung cancer. ${ }^{12} \mathrm{~A}$ robust phase II trial, as recommended by the Medical Research Council in its guidance for complex interventions, ${ }^{15}$ is needed as a first step towards achieving a similar goal for people with advanced heart disease.

The proposed study outlined here incorporates these issues using a mixed methods, phase I and II trials, design and is similar to methodologies used elsewhere to develop complex palliative care interventions for non-cancer illnesses. ${ }^{16}{ }^{17}$ The proposed trial is novel in that it includes a broad group of patients with CHF and ACS; it will assess whether well-validated clinical prognostic tools can be used to identify patients approaching EOL and will develop a feasible care planning intervention. In addition to assessing prognosis as a trigger, the study also seeks to explore the interface between acute cardiology services, primary care and specialist palliative care services.

Here we describe the findings of a qualitative interview study using patient-carer focus groups (PCFG) and a range of healthcare professionals (HCPs) to explore ways in which a holistic intervention could be tested in a randomised clinical trial setting.

\section{METHODS}

The basic design for a clinical trial outline was developed by the authors as part of a submission for research funding using their background knowledge and experience and based on other trial designs of a similar nature. ${ }^{1617}$ This was approved by the funder and by the local ethics committee on the understanding that the design of the study could be modified following a consultation/modelling phase which would involve focus groups of patients and carers and one-to-one interviews with a range of HCPs about the proposed trial design. PCFG were then undertaken with the members of an existing hospital-based heart failure patient-carer forum $(\mathrm{n}=7)$ and a second focus group was undertaken in conjunction with a local heart disease charity $(n=8$ participants) each lasting for $2 \mathrm{~h}$. Discussions were facilitated by an experienced qualitative researcher $(\mathrm{GH})$ using a set of questions, designed by the authors, addressing their experiences with clinical care and the proposed design of the randomised controlled trial (see online supplementary appendix 1A). Various options associated with the trial were presented and discussed with patients and carers including eligibility criteria and whether the control group should or should not receive EOL intervention.

A separate series of one-to-one interviews were conducted with a range of HCPs (total $\mathrm{n}=11$, palliative care consultant $n=3$, cardiology consultant $n=3$, heart failure specialist nurse $n=1$, medicine of the elderly specialist $n=1$, cardiology ward charge nurse $n=1$, general practitioner $n=1$, district nurse $n=1$ ) by an experienced qualitative researcher using a set of questions incorporating themes related to clinical care, palliative care and clinical trials design (see online supplementary appendix 1B).

Discussions regarding the proposed design of the trial were initially broad and later in the interview focused more on the eligibility criteria, types of patients that should be included and whether an active control group should be incorporated. Both types of participants were provided with a sample 'FCP' and a flow diagram of the basic proposed design of the clinical trial prior to the interviews. These documents acted as focal points for discussion. Interviews and focus groups were transcribed and analysed using NVivo to extract themes related to the rationale and design of a clinical trial of a holistic intervention addressing a range of issues related to EOL care.

The findings from these interviews and focus group discussions were then used to modify the proposed design of a trial of an intervention to support patients with advanced heart disease identified as being at high risk of death within the next 12 months. All participants gave signed informed consent. 


\section{RESULTS}

Patient's and carer's views

Care for cardiac patients

PCFG highlighted increasing difficulties associated with multiple care providers working in apparent isolation as a major difficulty in ensuring holistic care. From a PCFG perspective, care appears increasingly fragmented and ill designed to manage the needs of frail, elderly patients with multiple chronic conditions.

PCFG also identified the variation in access to specialist services as a key problem in providing holistic, patient-centred care. This applied in particular to heart failure nurse care where many services adhere to strict eligibility criteria which include left ventricular systolic dysfunction and recent hospital admission. Patients with access to the heart failure specialist nurse service were very appreciative of their support, but they expressed concern that this service was not available to everyone with heart failure and people with other types of advanced heart conditions.

PCFG welcomed the idea of future or anticipatory care planning, and appeared to recognise its value. However, a minority felt that this could be a very difficult process to engage in, expressing views that it needs to be carefully targeted and people should be able to choose, without pressure, not to engage in the process (box 1). PCFG highlighted the fact that some patients will already be well informed about their condition and its prognosis. However, it was also expressed that for those who have less insight into their condition, doctors and nurses should consider carefully how they will allay and minimise fears about engaging in a process of Future Care Planning.

\section{Box 1 Patient-carer views: fragmentation of care}

Once you get to our age, you discover that you've got more than one problem, and you see the various consultants who deal with the various problems, and they deal with you like a car. They put the carburettor right, they put the radiator right, but the holistic approach is missing (patient)

My condition is primarily a chronic lung condition but I also have a heart condition. So I have two separate areas of contact and they both know about each of the conditions but they're really only concentrating on the one they're dealing with, they soon forget, 'oh, you've got a heart condition, oh right!' And it's worrying particularly if you're being administered fairly serious medication and you've got to remember that you've got all these conditions (patient)

\section{Variation in access to specialist services}

We have a very good rapport and have chats with her (The Heart Failure Nurse). If there's something we don't understand, she's very good at explaining what's involved, so we're very happy (patient)

Having a nurse, it gives you a bit more confidence because you just know she's there. Everyone should have one, because it does make a heck of a difference (patient)
Proposed trial of Future Care Planning

There was a general consensus in the PCFG that the draft FCP planned for use in the trial (see online supplementary appendix 2) was comprehensive and addressed a number of concerns that families had about planning for the future (box 2). However, one carer made the point that a patient-centred anticipatory care plan must be flexible enough to accommodate those who are acting on behalf of their loved one possibly using power of attorney. Others suggested that it would be helpful to ensure that all contact details of the medical teams caring for a patient are included particularly for those with multiple comorbidities. One patient also questioned the appropriateness of asking patients to identify which potentially life-saving treatments they may or may not want.

PCFG were supportive of the proposal for a clinical trial and were satisfied with the basic design of the study. Opposing views about eligibility criteria were expressed by two participants in the same focus group with one indicating that eligibility should include people with advanced heart disease who were currently stable in the community and not necessarily those recently admitted to hospital. PCFG emphasised the need for families to be well informed and prepared before being approached about a trial testing the proposed intervention. Eligibility for the trial using a threshold value for estimated 12-month mortality was debated and discussed

Box 2 Patient-carer views: draft patient-held Future Care Plan (see appendix 2 "My Thinking Ahead Plan")

I have thought about all the questions (in the proposed patient-held Future Care Plan), and I think it's very, very good. Even for yourself to write down your thoughts and wishes. Everybody's wishes are different so therefore, if it's all written down and you've got this plan, I think, yes, it's very useful for the future (patient)

...personally, ignorance is bliss in some cases (patient)

What would worry me slightly about this, especially if you're filling it out on your own (Future Care Plan), is that suddenly an end, shall we say, opens up, the fact that you're filling in something that's to do with palliative care - ooh, a horrible word-I don't know if people with heart failure are taken through this before this or do they need to be sitting with a doctor or nurse who can take them through the fact that it's not as bad as it sounds. That would worry me...getting something like this and filling it in isolation, it's frightening (patient)

Well, if you follow all the questions, really I don't think you miss much at all. Because you're asking what people are interested in and what things they do at the moment and what they hope to continue, and in a way, I think that's very good for the professionals looking after us to know that-whether in fact you're doing anything or if you're doing nothing with your life, because I think it makes a big difference (patient) 
and a value of $20 \%$ was reached as one which would include a set of patients with a significantly higher than average mortality risk for cardiac patients. One further aspect of the final design of the trial which was discussed and agreed by the PCFG was whether to have a control group with no intervention or whether to have a group of patients where an intervention was provided but 12 weeks after discharge. It was generally agreed that it would be unethical not to provide an intervention of some sort to all patients who agreed to take part and so a delayed intervention group design was finally agreed (see figure 1).

\section{Views of HCPs}

EOL care for patients with heart disease

Irrespective of role, all HCPs who were interviewed identified the issue of managing the uncertainty of prognosis in people with advanced heart disease as a major challenge. Most agreed that prognostic uncertainty can cause HCP to prevaricate because they are worried about 'getting it wrong' (see box 3). They also expressed concerns that discussions about EOL could remove hope for the patient and their family.

\section{Proposed trial of Future Care Planning}

Several HCP participants drew a distinction between different types of patients with advanced heart disease and wanted more clarity about what type of patient would be eligible for the proposed trial. One cardiologist's view was that it would be relatively straightforward to recruit patients with coronary disease although it would be more complex to identify patients with heart failure since it is hard to pinpoint at which point in their illness trajectory they would become eligible (see box 4). For this group, one cardiologist suggested it may be useful to use repeated hospital admissions or functional status as a criterion for eligibility.

Care of the elderly physicians raised concerns about including patients in the trial with multimorbidity including those with cognitive impairment. Such patients are typically seen in acute medical takes and while they would be a group who may benefit considerably from Future Care Planning, they would be difficult to assess, recruit and retain in the proposed trial. However, this HCP stated that to omit these patients would be unfair and could miss a key opportunity.

There were no significant concerns raised with regard to using a clinical prognostic tool, such as the GRACE score, as a way of identifying patients for a palliative care intervention. However, it was highlighted by a number of HCPs that this approach has significant limitations and using such a tool in isolation may exclude many patients who could benefit from a Future Care Planning approach who have a high level of need and a low estimated 12-month mortality risk. In addition, it was highlighted that prognostic scores do not predict time to death nor do they accurately identify those who may benefit from a palliative care intervention.

Without exception, the HCPs we interviewed had no ethical concerns with a design utilising an early versus late intervention which they regarded as a standard approach for a trial (see figure 1).

Achieving and maintaining staff engagement and thinking ahead to what happens at the end of the trial were identified as important issues. In particular, HCPs identified the importance of keeping staff informed

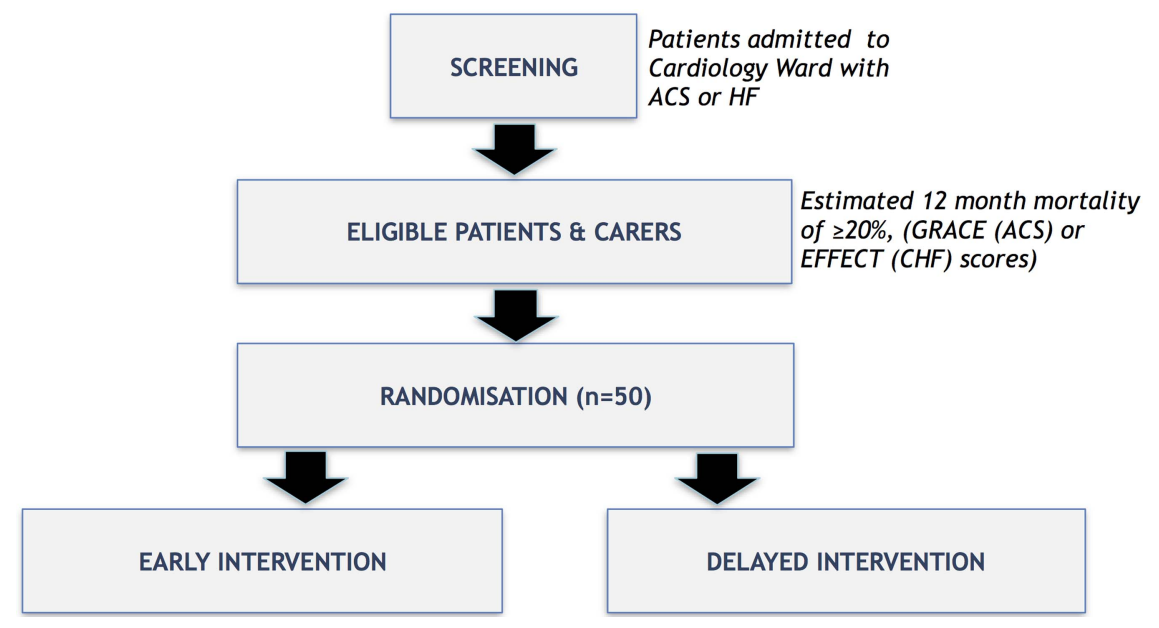

Figure 1 Adapted protocol for a randomised trial of Future Care Planning for patients with advanced heart disease: original flow diagram did not define the types of patients to be included, the threshold mortality risk for eligibility and the control group intervention. This final and agreed version identifies patients admitted to an acute cardiology ward with either acute coronary syndrome or heart failure and will be screened for eligibility-12-month mortality risk of $20 \%$ or greater at the time of discharge using the GRACE discharge score ${ }^{8}$ or the EFFECT score. ${ }^{7}$ Eligible patients need to survive to discharge and have capacity to consent for the study. Informal carers will also participate where identified by the patient. Proposed outcomes include quality of life assessed by questionnaire (EuroQoL-5D) and readmissions to hospital. Patients randomised to early intervention will be interviewed prior to discharge and those randomised to delayed intervention will receive the same interview 12 weeks following discharge. ACS, acute coronary syndrome; CHF, congestive heart failure; HF, heart failure. 
Box 3 Healthcare professional's views: prognostic uncertainty

...it's hard for health professionals to know where they (patients) are in their disease process, because we know they go up and down and they probably never come right back up to where they were the last time, but they're still functioning, and... at what point do you have that conversation? (District Nurse)

A lot of health professionals because of the trajectory of the disease and the up and down nature of it, nobody knows when the point of true palliation should kick in and people are very frightened because with some antibiotics or some steroids they could bounce back, not to the same state of health, each time declining and getting less well, but still not at the point where you would be comfortable saying, right, we're at the point of pure palliation (Palliative Care Consultant)

we've all seen patients who survive against the odds for a long time-if they outlive your expectations, that's OK... you might get the timing a bit wrong because you can't predict, but usually you are right that the decline has started (Heart Failure Specialist Nurse)

You don't want your patients to become obsessed and totally focused on their disease-(they've) got to get on with life as well (Medicine of the Elderly Consultant)

\section{Healthcare professional's views: risk of dying from a long-term condition}

"I don't think they see it, to the same extent as cancer patients-COPD patients as well. They (patients) see it as a limiting condition, it stops them doing things, it's not foremost in their mind that this is the thing they're going to die of" (District Nurse)

"Sometimes I think when it gets to the stage that you're doing DNAR forms.... it often comes as a big shock to either them or their family....it comes as a shock when they're told, 'we think this is it this time', because they've been in and out, bounced back and forward, got better, gone home" (Community Palliative Care Nurse)

about the trial, consideration of how the trial might dovetail with existing service developments and the importance of providing ongoing support beyond the trial period to participants who continue to require additional supportive care.

Hospital bed-days utilisation during follow-up was generally considered to be an appropriate outcome, although several expressed caution in interpreting what these data actually mean. Quality of life measures were also considered to be an appropriate outcome, although it was pointed out that these measures can also be difficult to interpret in this setting. Some HCPs suggested the inclusion of place of death and preference for place of death as outcome measures.

The initial study design of the clinical trial did not strictly define the types of patients that could be included, the eligibility criteria, the threshold mortality
Box 4 Healthcare professional's views: proposed trial of Future Care Planning

Patient selection for the trial:

One group is those with advanced heart failure for whom we already have some structure to post-discharge care through our HF nurse service and the second group....are those with end stage coronary disease, so these are patients with angina for whom there's not an awful lot more can be done for them by way of bypass surgery and invasive treatments, and where these patients are intermittently hospitalised when their angina reaches crisis point (Consultant Cardiologist)

A score based on a patient's functional status is useful because it identifies when quality of life is impaired to the extent that the patient needs more support (Consultant Cardiologist)

\section{Eligibility for the trial:}

...harder to put frail elderly patients into a protocol-driven trial because they are so different and they've got such a mix of co-morbidities and such a mix of drugs (Consultant in Medicine of the Elderly)

I think using (a cut-off of) 20\% (12 month mortality risk) is fair (Community Palliative Care Nurse)

It's still pretty high. If it's less than $20 \%$ people shouldn't imminently be dying so it gives you a chance to see what effect the intervention has (Consultant Cardiologist)

...So it's about identifying the point when you can have a reasonable conversation with somebody about deterioration, and is $20 \%$ (estimated mortality risk) right...If you make it higher you'll miss some people but you'll make the discussion more real and liveable, and that's your balance (Palliative Care Consultant)

A 20\% risk threshold would include lots and lots of frail elderly people. Many of them would have a 1 in 5 chance of dying within a year even without their heart failure. It's probably not an unreasonable threshold (Medicine of the Elderly Consultant)

\section{Trial outcome measures}

... if you're trying to prevent hospital admissions, if they're frequent fliers, then I would have thought they're the ones, the unstable ones. If you've been able to tweak something at home that prevented the admission, I suppose this is what this would do (District Nurse)

Obviously, you do have to look at bed days but ultimately they're spending more time in hospital, from their point of view...that's possibly better for them (Community Palliative Care Nurse)

...there's a subtle distinction, for example, between trying to measure differences in quality of life on a day to day basis, and measuring overall levels of comfort, security. (Medicine of the elderly consultant)

risk for inclusion and whether an intervention should be included for the control group. The final trial design (figure 1) represents a modified version taking account of the views of patients and HCPs interviewed in this 
study. This fundamental design of the proposed trial was similar to the original design presented to the PCFG and the HCPs during the interviews and focus groups. There was general agreement that a broad range of acute cardiac patients should be included and agreement was reached to include heart failure (with either reduced or preserved left ventricular systolic function) and patients with any ACS. There was discussion as to whether a delayed intervention was needed in the control group. Since current clinical services provided little or no EOL intervention for such patients, a final consensus, mainly driven by comments from patients and carers, was made to offer a delayed intervention to the control group given that the intervention addressed a clear need which was patient-centred and which could provide an apparent benefit to those who participated. The final component of the design which was agreed following the PCFG and HCP interviews was the threshold at which to set the 12-month mortality used as eligibility for the trial. The authors had proposed this to be somewhere between $20 \%$ and $40 \%$ and this was discussed by both groups. The final agreement of $20 \%$ was made largely by the PCFG after lengthy discussions as to what the typical mortality risk was for patients with CCF and ACS admitted acutely to hospital.

The findings from this modelling phase are currently being used to support the implementation of a phase II randomised clinical trial of a holistic intervention (figure 2) for patients with advanced heart disease.

\section{DISCUSSION}

This qualitative study examining patients', carers' and HCPs' views on the content and design of a clinical trial of Future Care Planning for patients with advanced heart disease has highlighted a number of important issues. The concerns raised by patients and carers regarding the current inadequate levels of care as cardiac patients approach EOL provide an important backdrop to the main theme of the work which was to seek their views on the content and design of a clinical trial. Patients and carers expressed views indicating that such a trial should redress the current inadequacies in a typical doctor-patient interaction which they felt had limited time and lacked a holistic approach particularly in cardiology outpatient clinic settings. The HCP participants highlighted the challenges in using meaningful selection criteria for the trial and the complexity of identifying precisely which component of any proposed intervention might influence outcomes. The findings of this work therefore reaffirm many of the findings of others in the field. ${ }^{18}{ }^{19}$ However, the novel aspect of the work is that the focus groups and interviews were extended beyond a general discussion stage to seek views on how the inadequacies in care could be redressed and a model developed which could be subsequently tested using a randomised trial approach.
Factors which might influence the outcomes which emerged from the PCFG discussions included the content and quality of the baseline or first discussion/ interview with the consultant, the content of the written FCP and ongoing support, for patient and carer, from a familiar HCP. The views from patients, carers and professionals indicated that a clinical trial should focus on providing adequate time to discuss the patient's current and future care needs and those of their carer, it should select patients on the basis of prognosis and needs, it should provide ongoing support with primary and secondary care working closely together to ensure good coordination of care and it should allow for adaptation of any care plan in a dynamic way that is aligned with the changing needs of the patients and their carers.

While this message is clear, delivering such a trial using non-palliative care physicians in an acute cardiology environment will be challenging. Finding the appropriate language to explain an uncertain prognosis is always difficult, ${ }^{20}$ and cardiologists with a firm culture of curative approaches may struggle to find that language. These challenges may delay the conversation until it is too late, or they may encourage the use of more vague, ambiguous or even contradictory language which can sometimes mislead the patient and their family or fail to communicate the seriousness of their condition adequately. Finding language that is balanced, caring and which makes sense of an uncertain future is one of the challenges of all palliative care even where the prognosis, good or bad, is more certain. However, the majority of the HCPs who we interviewed agreed that it should be possible in most cardiac patients with advanced disease. Surviving with a chronic condition that has an uncertain illness trajectory can mean that these patients, unlike patients with cancer, can reach a fairly advanced stage in their illness without realising that they have a condition that could and probably will cause their death.

Our findings have also highlighted a persisting tendency for patients and HCPs to associate palliative care with dying. This perception may prevent or discourage healthcare teams from offering palliative and supportive care to patients with significant symptom burden who may not have reached the end of their lives but who may benefit form additional supportive care. HCPs held the view that patients and carers may be reluctant to accept a form of support which they associate with EOL. This may reflect reluctance on the part of the HCPs as much as the patient. This is an important issue if we are to develop a model of integrating palliative care earlier in the illness trajectory of cardiac disease by the heart team caring for the patient. In addition to learning and developing the skills required to do this, these teams will also need to change attitudes and culture. Indeed, while this culture is increasingly acknowledged as important for patients with chronic heart failure, there is also a clear need for this approach in patients with other forms of advanced cardiac disease such as coronary and valvular heart disease. 


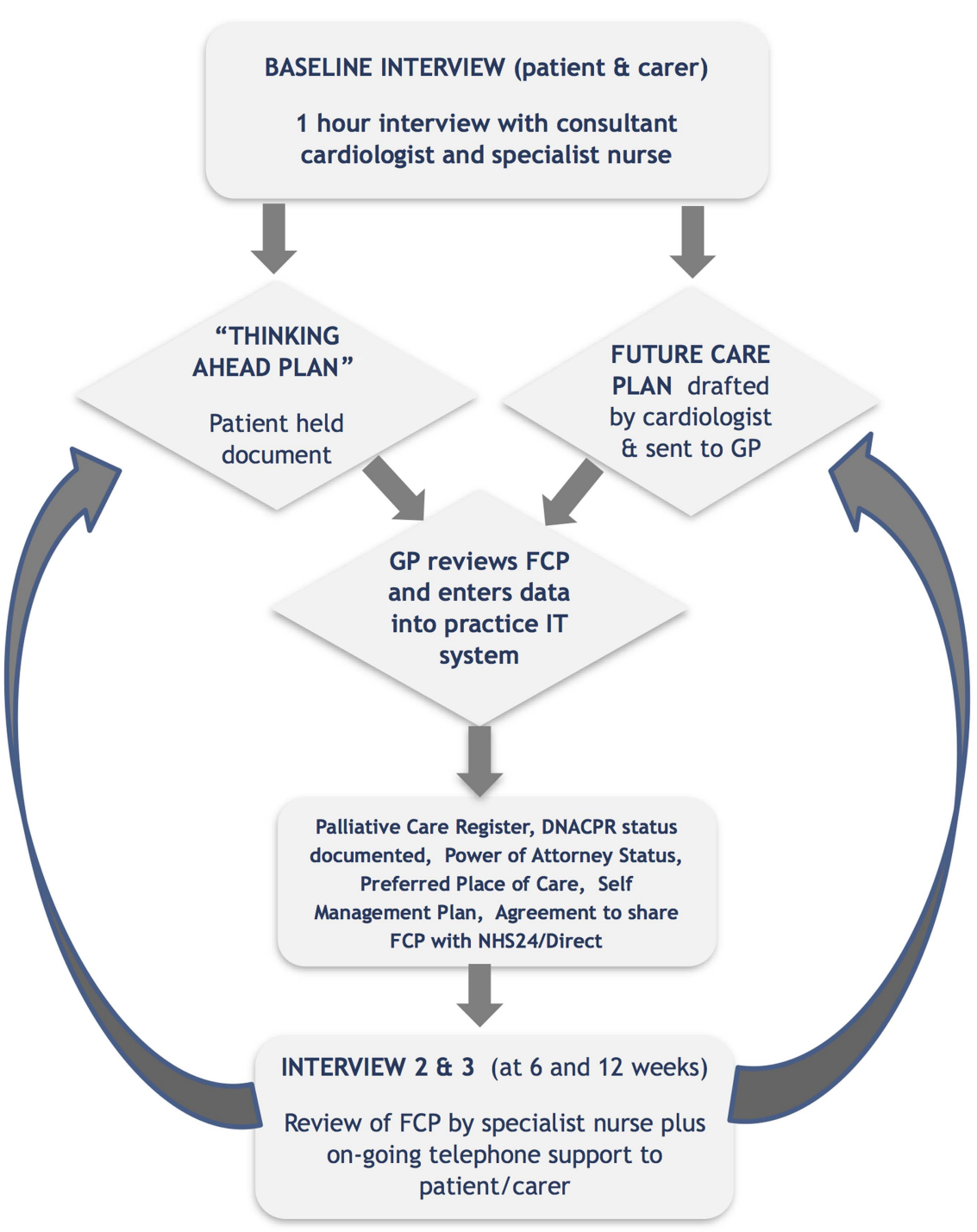

Figure 2 Future Care Plan (FCP) intervention: the intervention will last for 12 weeks. Patients randomised to early intervention will have a $1 \mathrm{~h}$ interview with a cardiologist prior to discharge where they will discuss their heart condition, other medical conditions and their concerns and plans for the future. The cardiologist, trained in Advanced Communication Skills, will aim to address a range of issues including (1) a FCP, agreed with the patient and their carer, which includes advice to healthcare professionals about what could and should be done if the patient's condition deteriorates once again, (2) whether the patient and their family have arranged Power of Attorney (or similar), (3) whether the patient wishes to consider the issue of DNACPR (Do Not Attempt Cardiopulmonary Resuscitation), (4) whether the patient wishes to express a preferred place of care should their condition deteriorate again, (5) whether the patient would consider being added to their general practitioner's (GP) Palliative Care register and (6) permission to share the content of the FCP electronically with out-of-hours medical services (NHS24/NHS Direct). Patients will also be encouraged to complete 'Thinking Ahead Plan', a locally developed patient-held anticipatory care plan (see online supplementary appendix 2). Patients randomised to delayed intervention will undergo the same interview 12 weeks after discharge. During the follow-up period of 12 weeks, the trial nurse will visit the patients/carers in their home at 6 and 12 weeks after the baseline interview in order to update the FCP with any changes and to review any DNACPR orders or make any necessary changes to the plan of care. An updated version of the FCP record will be communicated in writing to the GP at each of these time points. The nurse will be available to communicate with the patient by telephone at any time and will ensure optimal communication and coordination of care between GP, cardiologist, community-based nursing teams and palliative care teams (where appropriate).

The barriers to achieve good holistic care for patients nearing the end of their lives, well described in other settings, ${ }^{21} 22$ apply equally well in the acute cardiology setting. ${ }^{23}$ There is insufficient time to discuss such sensitive issues, the hospital environment is not ideal (particularly in multi-bedded rooms), cardiologists are not adequately trained and in cardiology there is a culture of doing more and never giving up. However, particularly in elderly patients and even with optimal interventions, the combination of CHF, coronary heart disease, valvular heart disease and other non-cardiac comorbidities is associated with poor prognosis. ${ }^{24} 25$ Indeed, the increasing 
use of transcatheter aortic valve implantation in patients who are deemed unsuitable for conventional surgery is increasingly recognised as a clinical challenge balancing aggressive intervention with supportive EOL care. ${ }^{26-29}$

Hence, patients with advanced heart disease and poor prognosis are clearly a target for better clinical care encompassing an approach that acknowledges that the patient may be nearing the EOL. These patients rarely receive care that addresses their individual needs and those of their informal carers. This care need not be labelled as palliative but can be delivered in the understanding that the future is uncertain and the risk of death, either sudden or with progressive symptoms, is significantly increased. This need not exclude a positive attitude to the patient's clinical care and where possible the HCP should emphasise the need for ongoing active and responsive care. The challenge, and arguably the key issue, is maintaining a positive attitude while simultaneously acknowledging a poor prognosis. However, if this approach can be adopted by the patients, their family, general practitioner and cardiologist, then it can potentially improve communication and understanding in a way that leads to better care without loss of hope for the patient. Any such intervention should be patientcentred in all aspects of its design and the impact on healthcare measures must be seen as secondary to the primary aim of improving quality of life. This is challenging even in cancer care where there have been few clinical trials adequately powered to show clinically meaningful benefits using a holistic approach. ${ }^{12}$ If improved clinical outcomes could be demonstrated in a clinical trial involving patients with advanced heart disease, then this would be extremely valuable to patients and the wider cardiology community.

This study has clearly demonstrated that patients, carers and HCPs have a number of concerns in relation to providing high-quality holistic care for patients with advanced heart disease. The approach reported here of seeking views on the inadequacies in service provision, designing an intervention model that could improve care and incorporating this into the design of a randomised trial is novel and important given the dearth of clinical trials in EOL care. The findings are currently being used to support implementation of a phase II randomised clinical trial of a holistic intervention involving Future Care Planning for patients with advanced heart disease.

\section{Author affiliations \\ ${ }^{1}$ Department of cardiology, Royal Infirmary of Edinburgh, Edinburgh, UK ${ }^{2}$ Department of Palliative Care, Royal Infirmary of Edinburgh, Edinburgh, UK ${ }^{3}$ Cardiology Department, Glasgow Royal Infirmary, Glasgow, UK ${ }^{4}$ Edinburgh Health Services Research Unit, Centre for Population Health Sciences, University of Edinburgh, Edinburgh, UK \\ ${ }^{5}$ Primary Palliative Care Research Group, Community Health Sciences- General Practice, University of Edinburgh, Edinburgh, UK}

Acknowledgements The authors wish to convey their gratitude to the patients, carers and healthcare professionals who contributed to the focus groups and interviews and to Marie Curie cancer Care for funding.
The support of Chest, Heart and Stroke, Scotland in organising the focus groups is also acknowledged.

Contributors MAD, KB and SM designed the study, analysed the findings and co-wrote the manuscript. GH facilitated and transcribed the interviews and analysed the data. SR, SC and KH helped interpret the findings. JR and AN supported the focus group work and helped interpret the findings of the study.

Funding This study was funded by Marie Curie Cancer Research (Project Grant A15867).

Competing interests None.

Ethics approval Lothian Research Ethics Committee.

Provenance and peer review Not commissioned; externally peer reviewed.

Data sharing statement No additional data are available.

Open Access This is an Open Access article distributed in accordance with the Creative Commons Attribution Non Commercial (CC BY-NC 3.0) license, which permits others to distribute, remix, adapt, build upon this work noncommercially, and license their derivative works on different terms, provided the original work is properly cited and the use is non-commercial. See: http:// creativecommons.org/licenses/by-nc/3.0/

\section{REFERENCES}

1. Department of Health. End of Life Care strategy, Promoting high quality care for all adults at end of life, 2008. http://www.dh.gov.uk/ prod_consum_dh/groups/dh_digitalassets/@dh/@en/documents/ digitalasset/dh_086345.pdf

2. Living and Dying well. Scottish Government, 2008. http://www. scotland.gov.uk/Resource/Doc/239823/0066155.pdf

3. Clinical Standards for Heart Disease, Health Improvement Scotland, 2010. http://www.healthcareimprovementscotland.org/programmes/ cardiovascular_disease/heart disease/heart disease standards. aspx

4. Haga K, Murray S, Reid J, et al. Identifying community based chronic heart failure patients in the last year of life: a comparison of the gold standards framework prognostic indicator guide and the Seattle heart failure model. Heart 2012;98:579-83.

5. Fenning S, Woolcock R, Haga K, et al. Identifying acute coronary syndrome patients approaching end-of-life. PLOS ONE 2012;7: e35536.

6. Iqbal J, Francis L, Reid J, et al. Quality of life in patients with chronic heart failure and their carers: a 3-year follow-up study assessing hospitalization and mortality. Eur J Heart Fail 2010;12:1002-8.

7. Lee DS, Austin PC, Rouleau JL, et al. Predicting mortality among patients hospitalized for heart failure derivation and validation of a clinical model. JAMA 2003;290:2581-7.

8. Pieper KS, Gore JM, FitzGerald G, et al. Validity of a risk-prediction tool for hospital mortality: the global registry of acute coronary events. Am Heart J 2009;157:1097-105.

9. Boyd K, Mason B, Kendall M, et al. Advance care planning for cancer patients in primary care: a feasibility study. $\mathrm{Br} J$ Gen Pract 2010;60:e449-58.

10. Boyd KJ, Murray SA, Kendall M, et al. Living with advanced heart failure: a prospective, community based study of patients and their carers. Eur J Heart Fail 2004;6:585-91.

11. Murray S, Boyd K. Using the 'surprise question' can identify people with advanced heart failure and COPD who would benefit from a palliative care approach. Palliat Med 2011;25:382.

12. Temel JS, Greer JA, Muzikansky A, et al. Early palliative care for patients with metastatic non-small-cell lung cancer. $N$ Engl J Med 2010;363:733-42.

13. Pellerin MA, Elwyn G, Rousseau M, et al. Toward shared decision making: using the OPTION scale to analyze resident-patient consultations in family medicine. Acad Med 2011;86:1010-18.

14. Grande GE, Todd CJ. Why are trials in palliative care so difficult? Palliat Med 2000;14:69-74. Review.

15. Campbell M, Fitzpatrick R, Haines A, et al. Framework for design and evaluation of complex interventions to improve health. BMJ 2000;321:694-6.

16. Sampson EL, Thuné-Boyle I, Kukkastenvehmas R, et al. Palliative care in advanced dementia; a mixed methods approach for the development of a complex intervention. BMC Palliat Care 2008;7:8.

17. Farquhar MC, Higginson IJ, Fagan $\mathrm{P}$, et al. The feasibility of a single-blinded fast-track pragmatic randomised controlled trial of a 
complex intervention for breathlessness in advanced disease. BMC Palliat Care 2009;8:9.

18. Barnes S, Gardiner C, Gott M, et al. Enhancing patient-professiona communication about end-of-life issues in life-limiting conditions: a critical review of the literature. J Pain Symptom Manage 2012;44:866-79.

19. Ryder M, Beattie JM, O'Hanlon R, et al. Multidsciplinary heart failure management and end of life care. Curr Opin Support Palliat Care 2011:5:317-21.

20. Maciasz RM, Arnold RM, Chu E, et al. Does it matter what you call it? A randomized trial of language used to describe palliative care services. Support Care Cancer 2013;21:3411-19.

21. Davies N, Maio L, Vedavanam K, et al. Barriers to the provision of high-quality palliative care for people with dementia in England: a qualitative study of professionals' experiences. Health Soc Care Community 2014;22:386-94.

22. Granek L, Krzyzanowska MK, Tozer R, et al. Oncologists' strategies and barriers to effective communication about the end of life. $J$ Oncol Pract 2013;9:e129-35.

23. Kavalieratos D, Mitchell EM, Carey TS, et al. "Not the "grim reaper service"': an assessment of provider knowledge, attitudes, and perceptions regarding palliative care referral barriers in heart failure. J Am Heart Assoc 2014;3:e000544.
24. Charlson ME, Pompei P, Ales KL, et al. A new method of classifying prognostic comorbidity in longitudinal studies: development and validation. J Chronic Dis 1987;40:373-83.

25. Radovanovic D, Seifert B, Urban $\mathrm{P}$, et al. Validity of charlson comorbidity index in patients hospitalised with acute coronary syndrome. Insights from the nationwide AMIS plus registry 2002-2012. Heart 2014;100:288-94.

26. Daly MJ, Monaghan M, Hamilton A, et al. Short-term efficacy of palliative balloon aortic valvuloplasty in selected patients with high operative risk. J Invasive Cardiol 2012;24:58-62.

27. Deutsch MA, Bleiziffer S, Elhmidi Y, et al. Beyond adding years to life: health-related quality-of-life and functional outcomes in patients with severe aortic valve stenosis at high surgical risk undergoing transcatheter aortic valve replacement. Curr Cardiol Rev 2013:9:281-94

28. Goldstein NE, May CW, Meier DE. Comprehensive care for mechanical circulatory support: a new frontier for synergy with palliative care. Circ Heart Fail 2011;4:519-27.

29. Lauck S, Garland E, Achtem L, et al. Integrating a palliative approach in a transcatheter heart valve program: bridging innovations in the management of severe aortic stenosis and best end-of-life practice. Eur $J$ Cardiovasc Nurs 2014:13:177-84. 